\title{
Decision-making in the Management of Badly Decayed First Permanent Molars in Children and Adolescents
}

\author{
El Meligy $\mathrm{OA}^{* 1}, \mathrm{Al}$ Nowaiser $\mathrm{AM}^{2}$, $\mathrm{Al}$ Sheikh $\mathrm{LA}^{3}$ and Caliwag $\mathrm{NO}^{3}$ \\ ${ }^{1}$ Professor of Pediatric Dentistry, Faculty of Dentistry, King Abdulaziz University, Saudi Arabia and Professor of \\ Pediatric Dentistry, Faculty of Dentistry, Alexandria University, Egypt \\ ${ }^{2}$ Associate Professor of Pediatric Dentistry, Faculty of Dentistry, King Abdulaziz University and Chairman of Intern- \\ ship Program at King Abdulaziz University, Saudi Arabia \\ ${ }^{3}$ General Practitioner, Faculty of Dentistry, King Abdulaziz University, Saudi Arabia
}

*Corresponding author: El Meligy OA, Department of Pediatric Dentistry, Faculty of Dentistry, King Abdulaziz University, Jeddah, Saudi Arabia, Tel: 0122871660, E-mail: omeligy@kau.edu.sa

Citation: El Meligy OA, Al Nowaiser AM, Al Sheikh LA, Caliwag NO (2016) Decision-making in the Management of Badly Decayed First Permanent Molars in Children and Adolescents. J Dent Oral Care Med 2(3): 302. doi: 10.15744/2454-3276.2.302

\section{Received Date: August 05, 2016 Accepted Date: August 29, 2016 Published Date: August 30, 2016}

\begin{abstract}
Aim: To report the decision making in management of badly decayed First Permanent Molars (FPMs) in children and adolescents among clinical consultants (specialists) and pediatric dentists at King Abdulaziz University (KAU), Faculty of Dentistry, Jeddah, Saudi Arabia.

Materials and Methods: An online survey was distributed to 150 clinical consultants (specialists) and pediatric dentists with 3 different clinical scenarios of badly decayed FPMs, in which the first age group of patients were from 6 to 9 years old, the second were 10 to 13 years old, and the third were 14 to 20 years old, the respondents were asked to choose only one proper treatment option from a list of 7 treatment choices for each clinical scenario.

Results: Overall response rate was $80 \%$. Regarding the first clinical scenario (deep caries is present in FPM with vital pulp), $47.5 \%$ of the respondent recommended indirect pulp capping for the first age group. While $52.5 \%$ of the respondents of the second age group and $55 \%$ of the respondents of the third age group recommended the use of a protective liner. Regarding the second clinical scenario (deep caries is present in the FPM exposing the pulp, however the tooth is still vital), $45.8 \%$ of the respondents recommended pulpotomy for the first age group, while $61.7 \%$ of the respondents of the second age group and $60.8 \%$ of the respondents of the third age group recommended direct pulp capping. Regarding the third clinical scenario (deep caries is present in the FPM exposing the pulp, however the tooth is non-vital), $70 \%$ of the respondents recommended apexification for the first age group. While $94.2 \%$ of the respondents of the second age group and $94.2 \%$ of the respondents of the last age group agreed to root canal treatment (RCT).
\end{abstract}

Conclusion: There are noticeable controversies among dentists when it comes to decision-making in management of badly decayed FPMs for 6 to 9 years old patients and an increased tendency towards agreement as the age of patients increases.

Keywords: Badly Decayed; First Permanent Molar; Pediatric Dentists; Decision-making

\section{Introduction}

The first permanent molar (FPM) is important for the development of normal occlusion. However, it erupts early into the oral cavity with little nutrition as it is still maturing, and so it is subjected to decay more often than the rest of the teeth [1]. In addition, the progression of decay of the FPM is most rapid during the first two years after eruption [2]. For the young patient population, the FPM has an extremely high caries rate. For instance, in China (2008) the decayed, missing, filled, teeth (DMFT) of FPMs were as high as $41 \%$ [3]. While in the United States (1980s) $50-60 \%$ of the FPM occlusal surfaces were decayed or restored by the age of 11-12 years. In Brazil the FPM DMFT was about 40\% and in the United Kingdom it was 45-48\% [4,5]. Whereas in Saudi Arabia, studies have revealed that the point prevalence of dental caries in FPM was recorded to be $66.4 \%$ with an average DMFT of $2.74 \pm 1.18$ concurrent with the finding that the prevalence increases consistently as age increased [6-8].

One of the first treatment choices of badly decayed FPM would be a simple conservative procedure of restorative filling. However, it is not always adequate when the tooth has become too weak or when the caries is too deep. Another issue arising in such cases is which restorative material to use during the procedure. Is it the metallic amalgam, which has the adequate mechanical and physical properties but poor esthetics? Or the resin composite restoration, which has adequate strength and esthetic properties? [9]. 
An important primacy in the treatment of deep caries lesions is preserving pulp vitality. This can be approached by either indirect or direct pulp capping [10]. Direct pulp capping is performed when there is a pinpoint exposure that occurred during caries removal, and the indirect pulp capping is done when the last layer of infected dentin is preserved to prevent pulp exposure. The capping materials used are calcium hydroxide $\left(\mathrm{Ca}(\mathrm{OH})_{2}\right)$ or mineral trioxide aggregate (MTA) as they have bacteriostatic effect. Some of the studies proved that it is a very successful procedure for few years, however other studies suggest that the cavity will still need to be reopened and it usually needs root canal treatment afterwards $[11,12]$. Stepwise caries excavation is considered one of the treatment scenarios, since this approach to caries excavation, as opposed to a direct complete excavation, would decrease the number of pulp exposures and accordingly enhance the possibilities for the pulp to heal [13].

Following carious exposure, the pulp can maintain its healing potential, as has been shown in several studies on asymptomatic and symptomatic permanent molars using MTA and calcium enriched mixture (CEM) cement as pulp-capping agents [14-17]. Vital pulp therapy is the treatment of choice in immature teeth with reversible pulpitis in which damage to the pulp and associated arrested root development occur as a result of carious pulp exposure [18]. In 2010, Nosrat and Asgary reported a case of an apexogenesis of a mandibular right second molar in a 12-year-old girl [19]. They have found that pulpotomy with a CEM cement allowed root development to continue.

When the decay reach the pulp, the treatment of choice is usually root canal treatment (RCT), however it is done only when there is an apical closure. This procedure is technique sensitive and the tooth will be weakened over time therefore it needs a full coverage restoration [20].

Extraction comes as an easy and convenient choice when treating a badly decayed tooth for children or young adults since the root of the tooth is not yet fully matured, but it is aggressive as well as complicated. Tipping and mesial drift of the second permanent molar will as an aftermath of extraction, so orthodontic treatment must be done to reposition second permanent molar and take the first permanent molar's place so that the normal occlusion may still occur. Some studies have come to an agreement that extraction of first permanent molar affects the eruption space of third permanent molar, reduces its impaction probability and it can result in occlusal relationships that are as good as those produced by premolar extraction therapy [21,22]. In 2010, Chen and Liggett directed a study to consider the various options for treatment and to look at the cost-benefit picture with respect to their long-term outcomes [2]. The findings of the study suggested that the decision to extract a FPM must be made in conjunction with the orthodontist who is ultimately responsible for the patient's comprehensive orthodontic/ orthopedic care. It appears that the following factors favor early FPM extractions: (i) Class I occlusion; (ii) premolar crowding; (iii) no missing permanent teeth; (iv) FPMs with poor treatment prognosis; and (v) dental age of 9-11. Even when these conditions are met, other conditions may prevent satisfactory space closure (such as ankylosis or primary failure of eruption of adjacent second permanent molars) [2]. However, not all patients can afford orthodontic treatment because its costly and time consuming.

\section{Problem Statement}

According to the literature, the FPM has early and very high rate of caries exposure, it almost challenging the vast majority of dentists. As simple as the case appears nevertheless the patients' compliancy, their young age, the lack of clear guidelines, the uncertainty of treatment outcomes place dentists in a state of confusion regarding the choice of management. In addition, it is usually affected by the knowledge and skills of clinical consultants in different dental departments and their long-term treatment plan. There are always controversies among consultants and general dentists when it comes to this matter.

\section{Justification}

Since there is a lack of studies and articles concerning this topic, as well as few to none argued this simple and common problem? Consequently, this research was conducted to enrich the literature and provide insight about the how clinical consultants and pediatric dentists manage badly decayed FPMs in children and adolescents. Besides it will point out the areas where further researches are in demand to be conducted.

\section{Materials and Methods}

\section{Aim}

To report the decision making in management of badly decayed FPMs in children and adolescents among clinical consultants (specialists) and pediatric dentists at King Abdulaziz University (KAU), Faculty of Dentistry, Jeddah, Saudi Arabia.

\section{Study design}

A cross-sectional study was conducted.

\section{Study population and location}

The study was carried out at KAU, Faculty of Dentistry, Jeddah, Saudi Arabia between August 2015 and August 2016. Selfadministered questionnaire was distributed online to 150 clinical consultants (specialists) and pediatric dentists working at KAU. 


\section{Reliability test}

To determine the questionnaire reliability, a test was done by distributing the questionnaire to 20 consultants working at KAU. After 2 weeks, a retest was done by distributing the same questionnaire to the same 20 consultants and comparing their responses (Test retest reliability).

\section{Data collection}

Data were collected through an online survey including sociodemographic information with three different clinical scenarios; the first clinical scenario (badly decayed FPM with vital pulp), second clinical scenario (badly decayed FPM with exposed vital pulp), and third clinical scenario (badly decayed FPM with exposed non-vital pulp), along with three age groups, the first age group of patients were from 6 to 9 years old with open apex and the second were 10 to 13 years old with closed apex, while the third were 14 to 20 years old with closed apex. The respondents were asked to choose only one proper treatment option from a list of 7 treatment choices for each clinical scenario: (1) Protective liner; (2) Indirect pulp capping; (3) Direct pulp capping; (4) Pulpotomy; (5) Apexification; (6) RCT; (7) Extraction.

\section{Sample size}

One hundred and twenty respondents (66 females and 54 males) have successfully completed the questionnaire. Uncompleted questionnaire was excluded from the study.

\section{Data analysis}

The obtained data were entered into the Statistical Package for Social Sciences (SPSS) version 23 software for OS X, 10.10 .5 operating system and were utilized for data analysis (Cross-tabulation and Chi square $(\mathrm{p}<.05)$ ).

\section{Ethical consideration}

Ethical approval was obtained from the Research Ethics Committee of the Faculty of Dentistry, KAU. The aim of the study was explained to all participants.

\section{Results}

The overall response rate was $80 \% ; 120$ respondents had successfully filled the survey out of 150 , in which 66 were females and 54 were males.

Regarding the first clinical scenario, the majority of the female respondents (31) (47\%) recommended a protective liner for the first age group, while the majority of male respondents (31) (57.4\%) recommended indirect pulp capping. For the second age group, a total of 63 (52.5\%) respondents recommended a protective liner, in which 34 (51.5\%) were females and 29 (53.7\%) were males. As for the third age group, a total of 66 (55\%) respondents recommended a protective liner, including 35 (53\%) females and 31 (57.4\%) males. There is an evidence of a significant difference between the patient's age (clinical status of the apex) and decisionmaking regarding the first clinical scenario $(\mathrm{P}=0.006)$ (Figure 1$)$.

\section{First Clinical Scenario.}

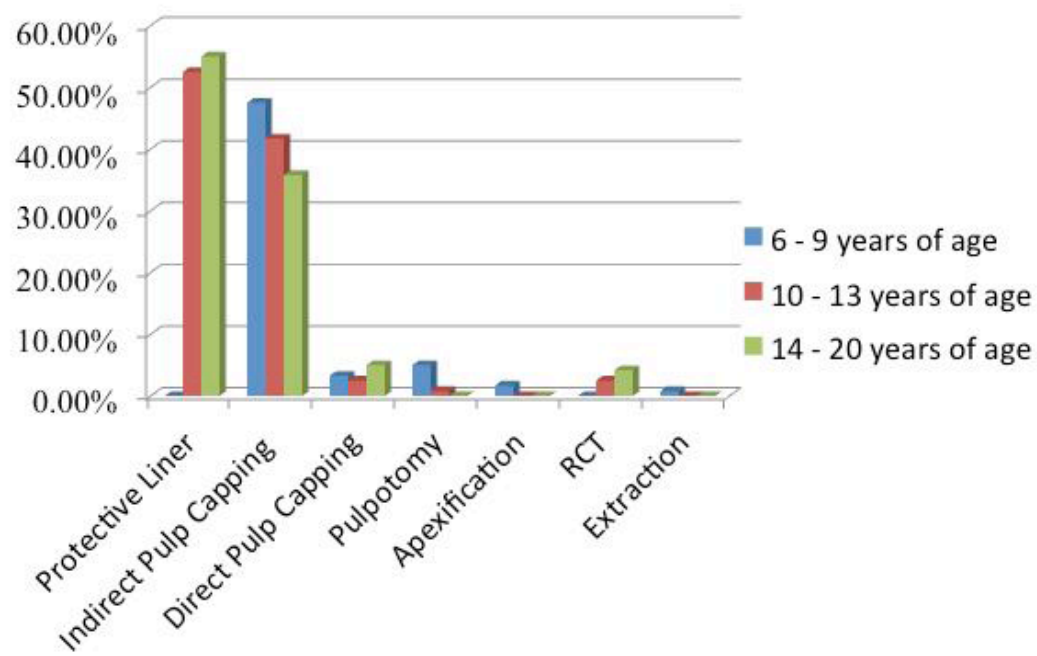

Figure 1: Evidence of a significant difference between the patient's age (clinical status of the apex) and decision-making regarding the first clinical scenario $(P=0.006)$ 
Concerning the second clinical scenario, the first age group, the majority of male respondents (30) (55.6\%) recommended direct pulp capping and the majority of female respondents (34) (51.5\%) recommended pulpotomy. The second age group, a total of 74 (61.7\%) respondents recommended direct pulp capping, in which 38 (57.6\%) were females and 36 (66.7\%) were males. Regarding the third age group, a total of $73(60.8 \%)$ respondents recommended direct pulp capping, in which 35 (53\%) were females and 38 (70.4\%) were males. There is an evidence of a significant difference between the patient's age (clinical status of the apex) and decision-making regarding the second clinical scenario $(\mathrm{P}=0.001)$ (Figure 2$)$.

\section{Second Clinical Scenario.}

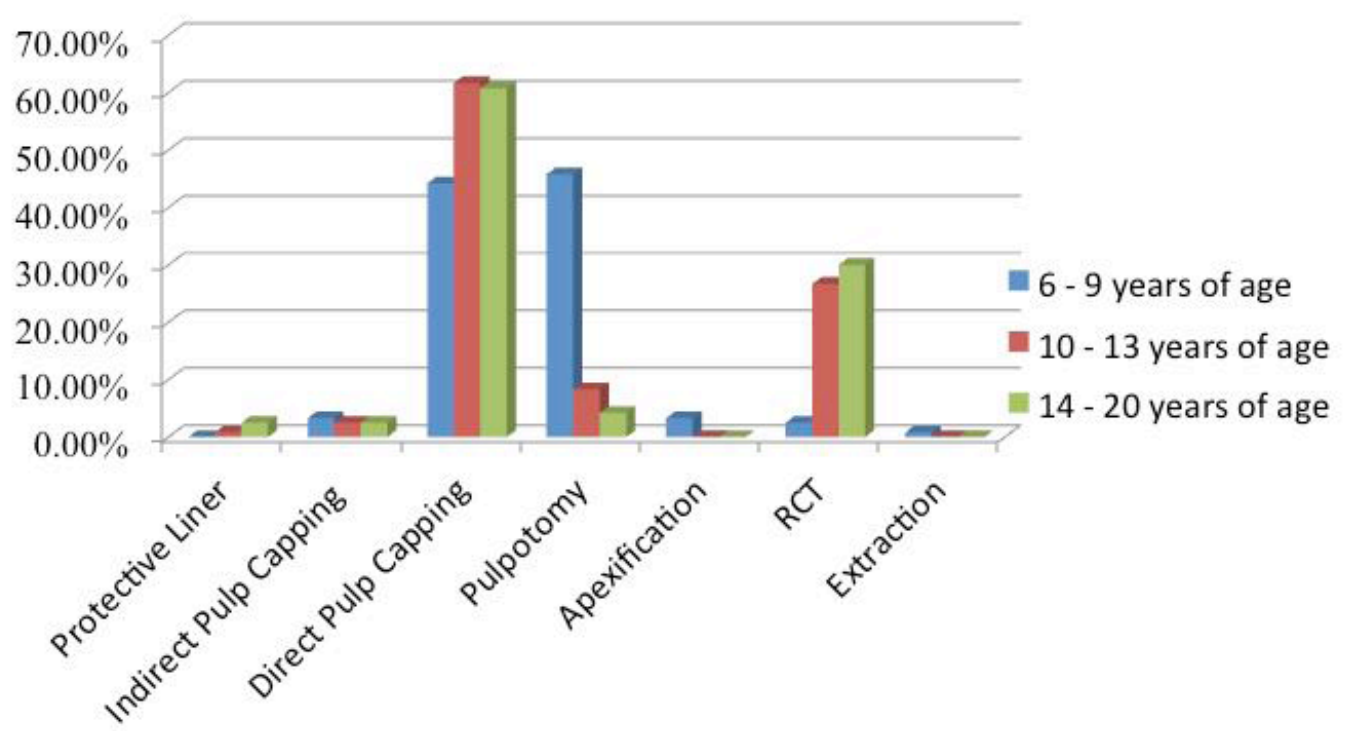

Figure 2: Evidence of a significant difference between the patient's age (clinical status of the apex) and decision-making regarding the second clinical scenario $(\mathrm{P}=0.001)$

With respect to the third clinical scenario, the first age group, a total of 84 (70\%) respondents recommended apexification, in which $51(77.3 \%)$ were females and $33(61.1 \%)$ were males. The second age group, a total of $113(94.2 \%)$ respondents recommended RCT, in which 62 (93.9\%) were females and 51 (94.4\%) were males. As regards the third age group, a total of 113 (94.2\%) respondents recommended RCT, in which 64 (97\%) were females and 49 (90.7\%) were males. There is an evidence of a significant difference between the patient's age (clinical status of the apex) and decision-making regarding the third clinical scenario $(\mathrm{P}=0.001)($ Figure $3)$.

Third Clinical Scenario.

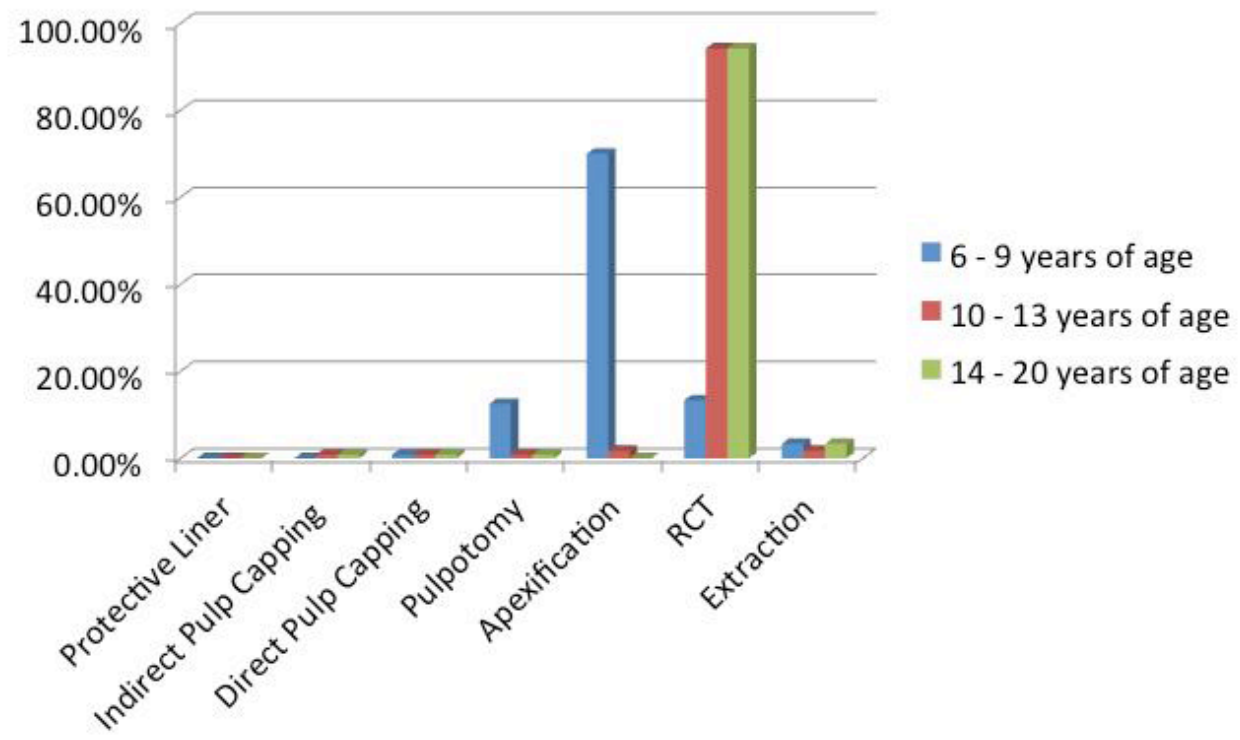

Figure 3: Evidence of a significant difference between the patient's age (clinical status of the apex) and decision-making regarding the third clinical scenario $(\mathrm{P}=0.001)$ 


\section{Discussion}

Dental treatment provided nowadays affects the overall health. Children and young adults who have not received early and adequate dental care and optimal systemic fluoride and lack adequate oral hygiene often develop deep carious lesions in the primary and permanent teeth which makes deep caries lesions a procedure of every day dental practice [23]. Clinical trials are usually concerned with evaluating the efficiency of clinical procedures, and are conducted under ideal research environment and conditions. However, the treatment of deep dentin caries is daily practice for most dentists worldwide. Nevertheless, there is no agreement in the dental profession regarding excavation depth, technique, or suitable diagnostic criteria or aids for caries removal. Although there are very limited data on how general practitioners treating deep caries, it can be assumed that complete removal of all affected biomass is common [24]. There is little known regarding the way dentists are adopting treatment decisions developed for management of badly decayed FPM. Hence, clinical scenario based surveys can be useful exploring the means dentists are making decisions and providing dental services in their routine based practice.

It is observable in this study that in the first age group (6-9 years old patient), in which the FPM tends to have an open apex, the respondents' recommendations were slightly inconsistent in all three clinical scenarios, regardless the vitality of the tooth and pulp exposure; which could be attributed to the lack of conclusive treatment guidelines in the literature.

In the present study, the vast majority of the respondents recommended a more conservative treatment such as a protective liner, and indirect pulp capping, for the first clinical scenario (deep caries is present in FPM with vital pulp). Moreover, it is reported that the majority of the respondents almost agreed to the same clinical treatment regarding the second clinical scenario in which they recommended direct pulp capping for both second and third age groups (10-13 and 14-20 years old). In addition, they tend to recommend apexification the first age group (6-9 years old) and RCT for the second and third age groups (10-13 and 14 -20 years old).

Findings of the present study reflect the conservative tendency within the included sample group. This could be attributed to the nature of their work place, since they are working in a university-based dental teaching hospital, which advocates preventive and conservative dentistry. Furthermore, a dentist's treatment decisions are thought to be influenced by variety of clinical and non-clinical patient and dentist factors [25]. Other factors suggested by the authors are the frequency of attendance at the dental clinic, the probability of treatment success and the risk/benefit relationship. The last is related to how much the benefits exceed the risks and the attitude of the patient and the dentist towards these risks. Also, the values both of the professional and the patient in relation to oral health, such as an esthetic preferences or attitudes based on the improvement of oral health; the threshold of the dentist with regard to taking the decision to intervene and the financial situation of the patient also influence the decisionmaking [26]. According to another study a post-qualification career of less than 10 years and attendance on postgraduate courses represents factors that prejudiced the most conservative clinical decision-making in the treatment of dental caries [27].

\section{Conclusions}

Vitality and exposure of the pulp, are considered additional clinical factors that affect the process of decision making for the management of badly decayed FMP. Furthermore, there are noticeable controversies among dentists when it comes to decision making in management of badly decayed FPM, especially for 6-9 years old patients and an increased tendency towards agreement as the age of the patient increases. The American Academy of Pediatric Dentistry (AAPD) guidelines are recommended to help dentists taking appropriate decision for the management of badly decayed FPMs in children and adolescents.

\section{Recommendations}

Further studies need to be conducted to investigate in depth the variety of factors affecting the process of decision making for the management of badly decayed FMP among dentists and the reliability of agreement among dentists for treatment recommendations. Also, future studies are needed to evaluate and explain the interactions between the multiple factors influencing the process of the decision making in treatment of badly decayed FPM. It would also be useful if the findings of this study are reproducible in the same region.

\section{References}

1. Moynihan P, Petersen PE (2004) Diet, nutrition and the prevention of dental diseases. Public Health Nutr 7: 201-26.

2. Chen JW, Leggitt VL (2012) Pulp treatment for young first permanent molars: To treat or to extract? Endod Topics 23: 34-40.

3. Cheng RB, Tao W, Zhang Y, Li Y (2008) Analysis of the first permanent molar caries epidemiological investigation in area of northeast China. Hua Xi Kou Qiang Yi Xue Za Zhi 26: 73-6.

4. Noronha JC, Massara Mde L, Souki BQ, Nogueira AP (1999) First permanent molar: First indicator of dental caries activity in initial mixed dentition. Braz Dent J 10: 99-104.

5. Albadri S, Zaitoun H, McDonnell ST, Davidson LE (2007) Extraction of first permanent molar teeth: results from three dental hospitals. Br Dent J 203: 408-9.

6. Zakirulla M (2012) Prevalance of first permanent molar caries among 7-10 years old school going boys in Abha City, Saudi Arabia. Bangladesh J Med Sci 11: 98-102. 
7. Al-Malik MI, Rehbini YA (2006) Prevalence of dental caries, severity, and pattern in age 6 to 7 -year-old children in a selected community in Saudi Arabia. J Contemp Dent Pract 7: 46-54.

8. Al-Samadani KH, Ahmad MS (2012) Prevalence of first permanent molar caries in and its relationship to the dental knowledge of 9-12-year olds from Jeddah, Kingdom of Saudi Arabia. ISRN Dent 2012: 391068. doi: 10.5402/2012/391068.

9. Qudeimat MA, Al-Saiegh FA, Al-Omari Q, Omar R (2007) Restorative treatment decisions for deep proximal carious lesions in primary molars. Eur Arch Paediatr Dent 8: 37-42.

10. Petrou MA, Alhamoui FA, Welk A, Altarabulsi MB, Alkilzy M, et al. (2014) A randomized clinical trial on the use of medical Portland cement, MTA and calcium hydroxide in indirect pulp treatment. Clin Oral Investig 18: 1383-9.

11. Al-Zayer M A, Straffon LH, Feigal RJ, Welch KB (2003) Indirect pulp treatment of primary posterior teeth: a retrospective study. Pediatr Dent 25: 29-36.

12. Leung RL, Loesche WJ, Charbeneau GT (1980) Effect of Dycal on bacteria in deep carious lesions. J Am Dent Assoc 100: 193-7.

13. Bjørndal L, Reit C, Bruun G, Markvart M, Kjældgaard M, et al. (2010) Treatment of deep caries lesions in adults: randomized clinical trials comparing stepwise vs. direct complete excavation, and direct pulp capping vs. partial pulpotomy. Eur J Oral Sci 118: 290-7.

14. Barrieshi-Nusair KM, Qudeimat MA (2006) A Prospective clinical study of mineral trioxide aggregate for partial pulpotomy in cariously exposed permanent teeth. J Endod 32: 731-5.

15. Witherspoon DE, Small JC, Harris GZ (2006) Mineral trioxide aggregate pulpotomies: a case series outcomes assessment. J Am Dent Assoc 137: 610-8.

16. Eghbal MJ, Asgary S, Baglue RA, Parirokh M, Ghoddusi J (2009) MTA pulpotomy of human permanent molars with irreversible pulpitis. Aust Endod J 35: 4-8.

17. Asgary S, Ehsani S (2009) Permanent molar pulpotomy with a new endodontic cement: A case series. J Conserv Dent 12: 31-6.

18. Witherspoon DE (2008) Vital pulp therapy with new materials: new directions and treatment perspectives-permanent teeth. J Endod. 34: S25-8.

19. Nosrat A, Asgary S (2010) Apexogenesis of a symptomatic molar with calcium enriched mixture. Int Endod J 43: 940-4.

20. Gudkina J, Mindere A, Locane G, Brinkmane A (2012) Review of the success of pulp exposure treatment of cariously and traumatically exposed pulps in immature permanent incisors and molars. Stomatologija 14: 71-80.

21. Bayram M, Özer M, Arici S (2009) Effects of first molar extraction on third molar angulation and eruption space. Oral Surg Oral Med Oral Pathol Oral Radiol Endod 107: e14-20.

22. Sandler PJ, Atkinson R, Murray AM (2000) For four sixes. Am J Orthod Dentofacial Orthop 117: 418-34.

23. Dean JA (2016) McDonald and Avery's dentistry for the child and adolescent (10th edn), Mosby, St. Louis: 221-42.

24. Oen KT, Thompson VP, Vena D, Caufield PW, Curro F, et al. (2007) Attitudes and expectations of treating deep caries: a PEARL Network survey. Gen Dent 55: 197-203.

25. Bader J, Shugars D (1992) Understanding dentists' restorative treatment decisions. J Public Health Dent 52: 102-10.

26. Kay EJ, Nuttall NM (1994) Relationship between dentists' treatment attitudes and restorative decisions made on the basis of simulated bitewing radiographs. Community Dent Oral Epidemiol 22: 71-4.

27. Traebert J, Marcenes W, Kreutz JV, Oliveira R, Piazza CH, et al. (2005) Brazilian dentists' restorative treatment decisions. Oral Health Prev Dent 3: 53-60.

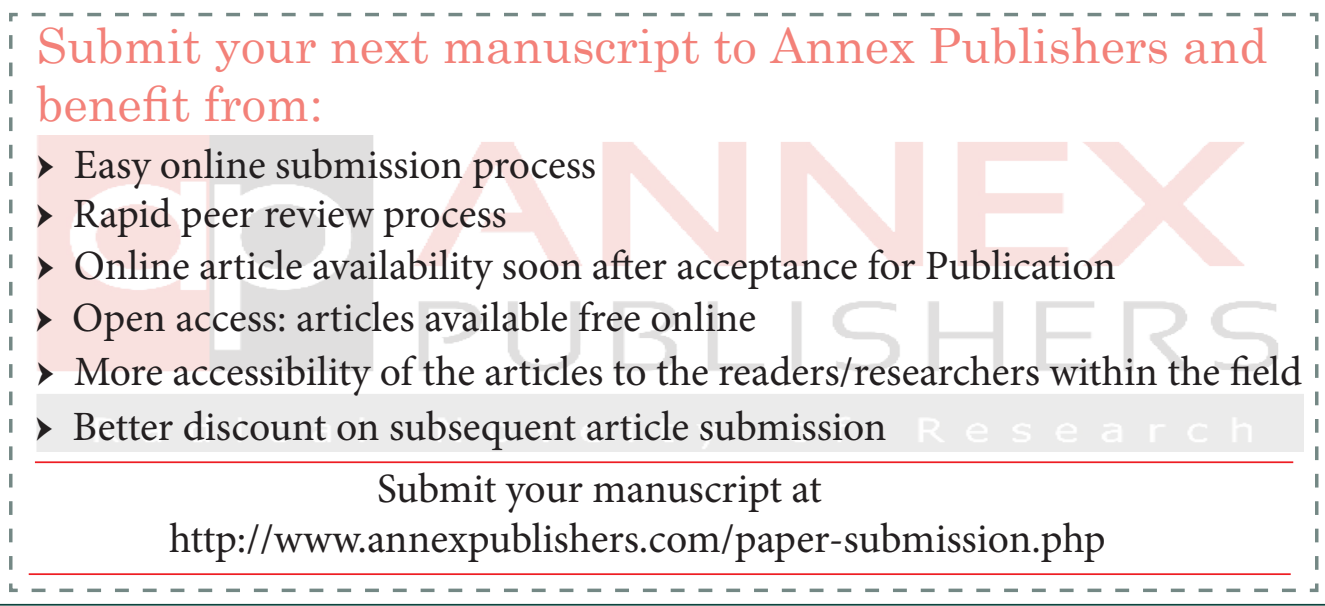

